Klaus Deimel, Alina Gerke \& Greta Molinski

\title{
Eine empirische Analyse der Bedeutung der strategischen Erfolgsfaktoren von Hidden Champions für mittelständische Unternehmen
}

Die Verlagsfassung der ZfKE ist verfügbar unter https://doi.org/10.3790/zfke.69.2.67

\section{Zusammenfassung}

Die Implementierung strategischer Erfolgsfaktoren rückt zunehmend in den Fokus kleiner und mittelständischer Unternehmen. Vor dem Hintergrund des überdurchschnittlichen Erfolgs sogenannter Hidden Champions (HC) stellt sich unter einer praxisorientierten Perspektive die Frage, welche Bedeutung mittelständische Unternehmen grundsätzlich den von Hermann Simon identifizierten Erfolgsprinzipien für HC für den Unternehmenserfolg zumessen. Die empirische Studie analysierte dazu die Bedeutung dieser Erfolgsfaktoren für mittelständische Unternehmen und untersuchte, ob Bedeutungsunterschiede zwischen erfolgreichen und weniger erfolgreichen Unternehmen der Stichprobe existieren. Im Rahmen einer explorativen, multivariaten Datenanalyse konnten außerdem zwei Cluster, die „Internationalen Innovatoren“ und die „Nationalen Traditionalisten“, im Datensatz identifiziert werden, die sich hinreichend in der Bedeutungszumessung der Erfolgsfaktoren voneinander unterschieden.

\begin{abstract}
The implementation of strategic success factors is increasingly gaining the attention of small and medium-sized enterprises. In light of the above-average success of so-called hidden champions (HC), the question arises which importance medium-sized companies in general attach to the success principles for HC identified by Hermann Simon. This empirical study analyzed the importance of these success factors for small and medium-sized enterprises and investigated whether differences in relevance exist between successful and less successful companies in the sample. An explorative, multivariate data analysis also identified two clusters in the data set, the "international innovators" and the "national traditionalists", which differed sufficiently from each other in the measurement of the importance of these success factors.
\end{abstract}

\section{Einleitung}

Strategisches Management ist schon lange keine Königsdisziplin von Großunternehmen mehr, sondern sollte zunehmend auch in den Fokus mittelständischer Unternehmen ${ }^{1}$ gerückt werden. Vor dem Hintergrund der heutzutage vorherrschenden, erhöhten Umweltdynamik sollten auch mittelständische Unternehmen stärker bestrebt sein, Erfolgspotenziale und Wettbewerbsvorteile systematisch zu identifizieren und weiterzuentwickeln. Diese Umweltdynamik tritt beispielsweise durch verkürzte Produktlebenszyklen, zunehmende Unsicherheit und Volatilität in Erscheinung. Zwar ist die Bedeutung der strategischen Unternehmensplanung auch in mittelständischen Unternehmen unumstritten, dennoch zeigt sich bislang ein deutliches Defizit hinsichtlich der tatsächlichen Umsetzung professioneller Ansätze zur Strategieimplementierung und -planung (Deimel et al. 2017). Dies kann mittel- bis langfristig die Erfolgschancen schmä-

\footnotetext{
${ }^{1}$ Definition gemäß des Deloitte.Mittelstandsinstuts der Universität Bamberg (Becker et al. 2008, 21). Für Erläuterungen siehe Seite 3.
} 
lern. Nichtsdestotrotz gibt es insbesondere in Deutschland mit den sogenannten Hidden Champions überdurchschnittlich erfolgreiche, mittelständische Unternehmen. Seit Anfang der 1990er Jahre hat sich Hermann Simon, der maßgeblich zur Erforschung der Hidden Champions beigetragen hat, mit der Untersuchung solcher überdurchschnittlich erfolgreichen Unternehmen des Mittelstands beschäftigt (Simon 1996; 2007; 2012). Damit hat er die heimlichen Weltmarktführer aus dem Mittelstand in den Fokus des öffentlichen Interesses gerückt. Doch hat das Thema nicht an Aktualität verloren - fast $50 \%$ der weltweiten Hidden Champions stammen aus Deutschland, so aktuelle Zahlen von Simon (vgl. iwd 2019).

Simon $(2012,83)$ bezeichnet diejenigen Unternehmen als Hidden Champions, die

1. die Nr. 1, 2 oder 3 auf dem Weltmarkt oder die Nr. 1 auf einem Kontinent sind.

2. einen Umsatz unter 5 Mrd. Euro realisieren;

3. nur einen relativ geringen Bekanntheitsgrad in der Öffentlichkeit besitzen.

Vor dem Hintergrund des teilweisen überdurchschnittlichen und nachhaltigen Erfolgs solcher mittelständischen Unternehmen stellt sich die Frage nach deren spezifischen Erfolgsfaktoren. So identifizierte Simon auf Basis einer fallstudienorientierten Vorgehensweise besondere Erfolgsfaktoren, die solche überdurchschnittlich erfolgreichen, mittelständischen Unternehmen auszeichnen (Knyphausen-Aufseß 1994; Simon 2007). Dabei sind Erfolgsfaktoren nach Rockart (1980, 49 ff.) solche Bereiche des Unternehmens, die das Erreichen der Unternehmensziele maßgeblich beeinflussen. Das Ziel der Erfolgsfaktorenforschung liegt somit darin, solche Faktoren zu identifizieren, die erfolgreiche Unternehmen von weniger erfolgreichen unterscheiden (Nicolai und Kieser 2002, 580 ff.).

Der vorliegende Beitrag greift den Hidden-Champions-Ansatz erneut auf und strebt mithilfe einer praxisorientierten, empirischen Untersuchung drei Ziele an. Erstens soll herausgefunden werden, welche Bedeutung mittelständische Unternehmen, die selbst keine Hidden Champions sind, den von Simon definierten Erfolgsprinzipien zumessen. Damit knüpft der Beitrag an Simons Hidden-Champions-Ansatz an, setzt aber einen anderen Fokus. Ziel ist es nicht, neue Erfolgsfaktoren zu definieren oder deren faktische Existenz erneut zu prüfen, sondern festzustellen, ob und in welchem Maße mittelständische Unternehmen die Relevanz dieser Erfolgsprinzipien für ihr eigenes unternehmerisches Handeln wahrnehmen. Ausgangspunkt der vorliegenden Untersuchung ist die Feststellung von Simon selbst, der in der Orientierung und Nutzung der strategischen Kernelemente der Hidden Champions erhebliche Verbesserungspotenziale nicht nur für Großunternehmen, sondern auch für Mittelständler sieht: ,Selbstverständlich können auch mittelständische Unternehmen, die weniger erfolgreich sind, von den Hidden Champions lernen. Sie sollten ihre eigenen Strategien mit denen der Hidden Champions vergleichen und feststellen, wo die Unterschiede liegen“" (Simon 2007, 42). Zusätzlich soll auch eruiert werden, ob die zahlreichen von Simon identifizierten Faktoren mit unterschiedlichen Gewichtungen in der Bedeutungszumessung seitens der befragten Mittelständler einhergehen.

Das zweite Ziel dieser Studie ist es, im Sinne der Erfolgsfaktorenforschung zu untersuchen, ob Unterschiede hinsichtlich der Bedeutungseinschätzung der Erfolgsfaktoren zwischen erfolgreichen und weniger erfolgreichen Unternehmen existieren. Diese Analyse soll Hinweise liefern, ob und inwiefern erfolgreiche Unternehmen andere Erfolgsfaktoren fokussieren als weniger bzw. nicht erfolgreiche Unternehmen.

Abschließend wurde eine multivariate, strukturentdeckende Clusteranalyse durchgeführt, deren Zweck darin bestand, Gruppen im Datensatz zu identifizieren, die die Bedeutung von 
Erfolgsfaktoren in sich möglichst homogen und im Vergleich zu anderen Gruppen möglichst heterogen einschätzen. Das dritte Ziel der Studie ist somit, zu untersuchen, ob sich spezifische Kombinationen von Erfolgsfaktoren identifizieren lassen, die auf unterschiedliche Geschäftsmodelle der Unternehmenstypen (Cluster) schließen. Außerdem soll der Frage nachgegangen werden, ob sich Unternehmen im Datensatz finden, die die Kernstrategien der Hidden Champions bereits aufgreifen und spezifischen Faktoren eine höhere Bedeutung zumessen als anderen.

Man beachte, dass bei der Zusammensetzung der Befragungsgrundlage bewusst von der KMU-Definition des IfM-Bonn oder der der Europäischen Union abgewichen wurde, um auch größere Unternehmen, die sich oftmals ebenfalls als mittelständische Unternehmen begreifen, in die Untersuchung einzubeziehen (vgl. Welter et al. 2014, 6, sowie die Erläuterungen zu den Teilgruppen des Mittelstandsuniversums inkl. gefühltem Nicht-Mittelstand und gefühltem Mittelstand ${ }^{2}$ in Welter et al. 2015, 41). Um uns nicht nur auf kleine und mittlere Unternehmen zu beschränken, sondern auch größere, mittelständische Unternehmen, die sich selbst noch als Mittelständler begreifen, aufnehmen zu können, haben wir uns für eine Quantifizierung an der Mittelstandsdefinition des Deloitte.Mittelstandsinstituts orientiert. Der Definition zufolge zählen zum Mittelstand sowohl Manager-geführte Unternehmen, die eine Mitarbeiterzahl von bis zu 3.000 Mitarbeitern und/oder eine Umsatzgröße von bis zu 600 Mio. Euro aufweisen, als auch Eigentümer- oder familiengeführte Unternehmen (Becker et al. 2008, 21). Ein weiterer Grund für die Abweichung von der KMU-Definition des IfM-Bonn ist neben der Diskrepanz zwischen gefühltem Mittelstand und gefühltem Nicht-Mittelstand auch die Tatsache, dass die Mittelstandsdefinition des Deloitte.Mittelstandsinstituts für die Zielsetzung der Studie als wesentlich praxisnäher und damit geeigneter angesehen wurde. So ist zu bedenken, dass die strategische Planung und Fokussierung von Erfolgsfaktoren eher bei größeren Unternehmen zu finden ist (Deimel et al. 2017, 97), weshalb der alleinige Fokus auf kleine und mittlere Unternehmen ohne den Mittelstand nicht zielführend für unsere Untersuchung gewesen wäre. Betrachtet man abschließend die Basiswerke von Simon zu den Hidden Champions, so wird auch hier klar, dass eine strenge Auslegung auf KMU bei der Erforschung der Erfolgsfaktoren nicht unbedingt zielführend ist. Simon gibt sogar eine Umsatzhöchstgrenze für Hidden Champions von 5 Milliarden Euro an (Simon 2012, 83), weil selbst Kleinstunternehmen mit wenigen Mitarbeitenden heutzutage global agieren können und die tatsächliche Größe mitunter zweitrangig ist. Natürlich zählen Unternehmen an der Höchstgrenze von 5 Mrd. Euro nach den üblichen Definitionen nicht mehr zu kleinen und mittleren Unternehmen, so Simon $(2012,84)$, sondern eher zu großen Mittelständlern. Gleichzeitig betont er aber auch:

„Wichtiger als die reine Größenabgrenzung ist für uns die Tatsache, dass diese Firmen im Zuge des starken Wachstums ihre typischen Eigenschaften, Strategien oder Führungsstile beibehalten haben. Die meisten Chefs dieser groß gewordenen Firmen achten darauf, die Stärken des Mittelständlers zu erhalten. (...) Die größten unserer Hidden Champions sind (...) große Unternehmen, die aber bei Weitem nicht an die globalen Top 500 heranreichen und in Deutschland nicht zu den Top 100 zählen. (...) In diesem Maßstab sind selbst die größten Hidden Champions allenfalls als mittelgroß einzustufen.“ $(2012,84)$

\footnotetext{
${ }^{2}$ Gefühlter Nicht-Mittelstand: Unternehmen, die sich selbst nicht als Mittelständler begreifen, aber rein formal welche sind. Gefühlter Mittelstand: Unternehmen, die sich selbst als Mittelständler begreifen, aber rein formal keine sind (Welter et al. 2015, 41).
} 
Vor dem Hintergrund der genannten Erläuterungen wurde eine bewusste Abweichung der KMU-Definition des IfM-Bonn angestrebt und die Deloitte-Mittelstandsdefinition für die vorliegende Untersuchung der Bedeutung der von Hidden Champions abgeleiteten Erfolgsfaktoren herangezogen. Damit wurden in der Untersuchung im Vergleich zur KMU-Definition größere Unternehmen aber im Vergleich zu den Hidden Champions immer noch Unternehmen mit moderater Größe betrachtet.

\section{Erfolgsfaktoren der Hidden Champions}

Hidden Champions unterscheiden sich von anderen Unternehmen dadurch, dass sie weitgehend unbemerkt von der öffentlichen Aufmerksamkeit durch die Wahl einer geeigneten Unternehmensstrategie ein überdurchschnittliches Wachstum und einen überdurchschnittlichen Erfolg aufweisen können. So definieren Simon (1996; 2007; 2012), Welter et al. (2014) und Blommen und Bothe (2007) in ihren Veröffentlichungen verschiedene Erfolgsfaktoren solcher Hidden Champions. In Tabelle 1 sind die für diese Studie zentralen Erfolgsfaktoren zusammen mit einer Kurzbeschreibung synoptisch aufgeführt.

\section{Methode}

Nachfolgend werden das Erhebungsdesign, die verwendeten Messinstrumente und die Stichprobe genauer beschrieben.

\section{Erhebungsdesign}

Zur Überprüfung der Frage, ob und inwieweit mittelständische Unternehmen diesen Erfolgsfaktoren in der Praxis Bedeutung zumessen, wurde eine empirische Untersuchung, basierend auf einem vorliegenden Datensatz mittelständischer Unternehmen in NRW des Wirtschaftsinformationsdiensts Creditreform/lexis nexis, durchgeführt. Unter der plausiblen Annahme, dass sich die Bedeutungszumessung von Erfolgsfaktoren durch mittelständische Unternehmen in NRW nicht signifikant von der Einschätzung von Unternehmen in Gesamtdeutschland unterscheiden, wurden zur Untersuchung der Bedeutung der Erfolgsfaktoren exemplarisch Mittelständler aus NRW befragt. Dabei wurde auf den sog. key informant approach (z. B. Marshall 1996, 92) zurückgegriffen, nach dem die Inhaber oder Geschäftsführer der entsprechenden Unternehmen zur Befragung herangezogen wurden. Grundlage der Erhebung bildete eine Adressdatenbank, die vom Wirtschaftsinformationsdienst Creditreform/lexis nexis zur Verfügung gestellt wurde, mit Unternehmen in der Größenordnung von bis zu 400 Mio. Euro Umsatz und einer Mitarbeiterzahl von bis zu 5.000 Mitarbeitern. Das Deloitte.Mittelstandsinstitut führt in seiner Definition als quantitative Kriterien des Mittelstands Größenklassen von bis zu 3.000 Mitarbeitern und bzw. oder bis zu 600 Mio. Euro Umsatz an. Es muss damit kritisch angemerkt werden, dass es keine hundertprozentige Passung zwischen klassischen Mittelstandsdefinitionen und den Größenklassen der zugrundeliegenden Datenbank in Hinblick auf die Mitarbeiterzahl gab. So können unter den Befragten auch Unternehmen sein, deren Mitarbeiterzahl zum Befragungszeitpunkt über 3.000 Mitarbeitern lag. Da es sich bei den quantitativen Bedingungen der Mittelstandsdefinition allerdings um eine ,und/oder Verknüpfung“ handelt, wird die Definition des Deloitte.Mittelstandsinstituts trotzdem gewahrt, die maximale Grenze von 600 Mio. Euro Umsatz in keinem Fall überschritten wurde. 
Manuskript - Hidden Champions: Eine empirische Analyse für mittelständische Unternehmen

Tabelle 1: Ausgewählte Erfolgsfaktoren von Hidden Champions (HC)

\begin{tabular}{|c|c|}
\hline Erfo & \\
\hline $\begin{array}{l}\text { Enge Defini- } \\
\text { tion des Kern- } \\
\text { geschäfts }\end{array}$ & $\begin{array}{l}\text { - HC fokussieren sich auf Nischenmärkte, in denen sie international oder global eine markt- } \\
\text { führende Stellung erobert haben (Hamel und Prahalad 1994, } 79 \mathrm{ff} \text {.) } \\
\text { - HC weisen ein enges, spezialisiertes Leistungsangebot auf und sind damit eher tief als } \\
\text { breit aufgestellt }\end{array}$ \\
\hline $\begin{array}{l}\text { Frühzeitige } \\
\text { und starke In- } \\
\text { ternationali- } \\
\text { sierung im } \\
\text { Kernsegment }\end{array}$ & $\begin{array}{l}\text { - HC realisieren Wachstum häufig durch eine starke internationale oder globale Expansion } \\
\text { im Kernsegment } \\
\text { - HC drängen oft früh auf internationale Märkte, um Pionierprämien zu nutzen und eine } \\
\text { starke Marktstellung aufzubauen } \\
\text { - Kunden weisen homogene Bedürfnisse auf, weshalb die Strategie auf weltweite Kunden } \\
\text { leicht übertragbar ist }\end{array}$ \\
\hline $\begin{array}{r}\text { Kund } \\
\text { fisch } \\
\text { tungs } \\
\text { uno } \\
\text { Kunc } \\
\text { d }\end{array}$ & $\begin{array}{l}\text { itarbeitende auch das Top-Management hält regelmäßig Kontakt zu den Kun- } \\
3 \text { um deren Bedürfnisse und Anforderungen } \\
\text { sebot stark durch den Kunden geprägt und oft gemeinsame Produktentwick- } \\
\text { oller } 2006,1067 \mathrm{f} \text {.) } \\
\text { d damit auf einem hohen technischen Niveau, genau auf den Kunden zuge- } \\
\text { d durch kundenspezifische Serviceleistungen abgerundet } \\
\text { öhte Kundenbindung und Transaktionskosten für Wechsler, höhere } \\
\text { sbarrieren für Wettbewerber (vgl. Simon } 2007,176 \text { f.) }\end{array}$ \\
\hline $\begin{array}{l}\text { Qu } \\
\text { statt } \\
\text { rers } \\
\text { str }\end{array}$ & $\begin{array}{l}\text { rerhältnis } \\
\text { Größe der HC oft nicht mög- }\end{array}$ \\
\hline $\begin{array}{l}\text { ations- } \\
\text { rschaft }\end{array}$ & $\begin{array}{l}\text { ler Erfolgsfaktor der HC sind ihre Innovationen und ihre Innovationsschnelligkeit } \\
\text { atente setzten viele HC eher auf ihren permanenten Innovationsprozess im Unter- } \\
\text { en } \\
\text { nung und Entwicklung ist eine wesentliche Quelle der Wettbewerbsvorteile von HC }\end{array}$ \\
\hline $\begin{array}{l}\text { Organisati- } \\
\text { onsstruktur } \\
\text { und Wert- } \\
\text { schöpfungs- } \\
\quad \text { tiefe }\end{array}$ & $\begin{array}{l}\text { - HC setzen auf einfache Organisationsstrukturen und direkte Kommunikationswege. Inter- } \\
\text { national tätige HC stellen ihre Organisation oft von einer funktionalen auf eine divisionale } \\
\text { um (vgl. Simon 2007, } 262 \text { ff.) } \\
\text { - Hohe Fertigungs- und Wertschöpfungstiefe bei den Kernkompetenzen aus Gründen der } \\
\text { besseren Kontrollierbarkeit der Qualität und Schutz der Technologie (vgl. Simon 2007, } \\
272 \text { ff, } 280 \text { ff.) }\end{array}$ \\
\hline $\begin{array}{l}\text { Finanzkraft } \\
\text { und Kosten- } \\
\text { management }\end{array}$ & $\begin{array}{l}\text { zkraft aus } \\
\text { vgl. u.a. Myers 1984, } 576 \text { f.) der Finan- } \\
\text { Bankfinanzierung } \\
\text { g } \\
\text { lig für konjunkturzyklische Schwankun- }\end{array}$ \\
\hline $\begin{array}{l}\text { Mitarbeiter- } \\
\text { motivation } \\
\text { und Mitarbei- } \\
\text { terqualifika- } \\
\text { tion }\end{array}$ & $\begin{array}{l}\text { - Hohe Mitarbeitermotivation und -qualifikation, niedriger Krankenstand und Loyalität zum } \\
\text { Unternehmen sind für den Erfolg von HC unabdingbar } \\
\text { - HC zeichnen sich durch eine Höchstleistungskultur aus (zur Unternehmenskultur und wir- } \\
\text { tschaftlichem Erfolg siehe auch Rosenstiel 2009, } 28 \mathrm{ff} \text {.) } \\
\text { - Rekrutierung hochqualifizierter Mitarbeitender aufgrund der geringen Größe und Be- } \\
\text { kanntheit von HC erschwert } \\
\text { - Mitarbeitende zentrale Quelle für Innovation und Erfolg }\end{array}$ \\
\hline $\begin{array}{l}\text { Führungsper- } \\
\text { sönlichkeiten } \\
\text { und Füh- } \\
\text { rungsstil }\end{array}$ & $\begin{array}{l}\text { fig eigentümergeführt und daher zumeist stark durch die Wertvorstellungen und die } \\
\text { rungspersönlichkeit der Eigentümer geprägt } \\
\text { rung in HC gezeichnet von intensiver Identifikation mit der Aufgabe, einer fokussier- } \\
\text { „Zielstrebigkeit““, „Furchtlosigkeit““ und einer starken „Energie und Motivationsfähig- } \\
\text { rung häufig ambivalent, autoritär in den Grundwerten und partizipativ im Detail } \\
\text { gfristorientierung und hohe Führungskontinuität (vgl. Simon 2007, } 361 \text { ff.) } \\
\text { e Umsetzungskompetenz bei Entscheidungen und Projekten }\end{array}$ \\
\hline
\end{tabular}


Die Rücklaufquote der insgesamt 2.000 ausgesendeten Fragebögen betrug 5,8 \%, sodass eine Gesamtzahl von insgesamt $N=116$ Fragebögen verarbeitet werden konnte. Aufgrund der vergleichsweise geringen Rücklaufquote sind die Ergebnisse der Studie jedoch eher als exploratorisch einzuschätzen, denn als repräsentativ. Um einen höchstmöglichen Stichprobenumfang zu erhalten, wurden auch unvollständig ausgefüllte Umfragen berücksichtigt. Somit wurde jedes Item mit einem eigenen Stichprobenumfang ausgewertet, weshalb es im Verlauf der Auswertungen zu variierenden Stichprobengrößen kommen kann.

\section{Struktur des Fragebogens}

Auf Basis der Werke von Simon (1996; 2007; 2012), Welter et al. (2014) und Blommen und Bothe (2007) wurden analytisch insgesamt 20 Erfolgsfaktoren identifiziert, deren genaue Operationalisierung Tabelle 2 zu entnehmen ist. Für eine bessere Übersicht wurden die 20 Erfolgsfaktoren den fünf Oberkategorien „Produkt/Services“, „Marketing/Vertrieb“, „Management“, „Finanzen/Betriebswirtschaft“ und „Mitarbeiter“ zugeordnet. Die Zuordnung erfolgte auf Basis von Plausibilitätsüberlegungen sowie der inhaltlichen und systematischen Passung der Erfolgsfaktoren zu den Oberkategorien. Die Oberkategorien sind somit als Oberbegriffe zu verstehen. Die befragten Unternehmen wurden gebeten, die Bedeutung dieser Erfolgsfaktoren auf einer fünfstufigen Likert-Skala ( 1 = sehr geringe/keine Bedeutung bis 5 = sehr hohe Bedeutung) für ihr Unternehmen einzuschätzen.

Der Fragebogen umfasste neben diesen geschlossenen Items zu den verschiedenen Bereichen der strategischen Erfolgsfaktoren ebenfalls Items für die Erhebung der Strukturdaten der befragten Unternehmen (Anzahl der Mitarbeitenden, Branche, Eigentümerstruktur), die eine tiefergehenden Analyse des Datensatzes erlauben. Darüber hinaus wurde der Erfolg der befragten Unternehmen erhoben. Aufgrund der hohen Sensibilität mittelständischer Unternehmen bei diesen Daten wurden hierbei keine absoluten Werte oder konkrete Zahlen erfragt, sondern mittels einer Selbsteinschätzung die Position des eigenen Unternehmens im Vergleich zum Branchendurchschnitt (über Branchendurchschnitt, Branchendurchschnitt, unter Branchendurchschnitt) erfragt.

Die Messung des Unternehmenserfolgs stellt ein wesentliches Konstrukt innerhalb der Entrepreneurship-Forschung als auch insbesondere im Kontext von mittelständischen Unternehmen dar. Obwohl Unternehmenserfolg einen der Kernbegriffe der Betriebswirtschaftslehre darstellt, gibt es bisher keinen gemeinsamen Konsens hinsichtlich der Methodik der Erfolgsmessung. Dies zeigt sich unter anderem in Studien zur Erfolgsfaktorenforschung durch die Verwendung unterschiedlicher Erfolgsmaßstäbe, die die Vergleichbarkeit der Ergebnisse der Studien begrenzen. „Um sich diesem Phänomen zu nähern, bedarf es zunächst seiner Konzeptualisierung, d. h. ,Erfolg' wird als abstrakte und nicht direkt messbare Größe, also als Konstrukt, verstanden, das unter Heranziehung von theoretischen Erklärungsansätzen inhaltlich und semantisch konkretisiert wird.“ (Evanschitzky 2003, 54). Um eine solche latente Variable erfassen zu können, müssen beobachtbare Indikatoren abgeleitet werden.

Inhaltlich stehen der Betriebswirtschaftslehre eine Vielzahl von Erfolgsindikatoren zur Verfügung. Diese reichen von harten Finanzkennzahlen (z. B. Rentabilität, Aktienkurse, Gewinn, Tobin's Q) über Marktkennzahlen (z. B. Marktanteil, Absatzwachstum, Umsatz, Markenwert) hin zu weichen Erfolgsindikatoren (z. B. Zufriedenheit mit dem Unternehmenserfolg). Darüber hinaus lässt sich weiterhin feststellen, dass sich die Zielerreichung des Unternehmens häufig in 
Abhängigkeit davon unterscheidet, ob die Beurteilung durch die Eigentümer oder das Management bzw. eher stakeholderorientiert durch Arbeitnehmer, Kunden, Lieferanten oder sonstige gesellschaftliche Anspruchsgruppen erfolgt. Welge et al. (2017, 226 f.) fokussieren in Zusammenhang mit der Erfolgsfaktorenforschung vorwiegend auf jahresabschluss- bzw. finanzorientierte Erfolgskennzahlen, wie klassische Gewinn- bzw. Rentabilitätsgrößen bis hin zu Konzepten wertorientierter Zielgrößen. „Einer Auswertung von Ambler/Kokkinaki (1997) zufolge wurden in Erfolgsfaktorenstudien am häufigsten der Umsatz (22,3 \%), der Marktanteil (17,1 $\%$ ), der Gewinn (11,0\%) und die Markenwahl/Kaufintention (11,0\%) verwendet“" (Baumgarth und Evanschitzky 2009, 243).

Tabelle 2: Untersuchte Erfolgsfaktoren in der empirischen Analyse

\begin{tabular}{|c|c|c|}
\hline Kategorie & Item & Bezeichnung \\
\hline $\begin{array}{l}\text { Produkt/ } \\
\text { Services }\end{array}$ & $\begin{array}{l}\text { - } \text { Einzigartige Geschäftsidee } \\
\text { - Hervorragende Produkt-/Servicequalität; bestmögliche } \\
\text { Befriedigung der Kundenbedürfnisse } \\
\text { Konzentration auf Nischenmärkte; enges spezialisier- } \\
\text { tes Leistungsspektrum (eher tiefes als bereites Leis- } \\
\text { tungsprogramm; geringe Produktdifferenzierung) } \\
\text { - F\&E als Kernkompetenz; hohe technologische Innova- } \\
\text { tivität; Technologieführerschaft }\end{array}$ & $\begin{array}{l}\text { - Geschäftsidee } \\
\text { - Produkt-/Servicequalität } \\
\text { - Nischenmärkte } \\
\text { - F\&E als Kernkompetenz }\end{array}$ \\
\hline $\begin{array}{c}\text { Marketing/ } \\
\text { Vertrieb }\end{array}$ & $\begin{array}{l}\text { - Gemeinsame Produktentwicklung mit Kunden } \\
\text { - Starke internationale bzw. globale Vermarktung der } \\
\text { - Unternehmensleistung } \\
\text { - } \text { Hohe Investitionen in Marketingmaßnahmen } \\
\text { - Hohe Kundennähe; lang andauernde, intensive und un- } \\
\text { - Günstige Produktpreis, Kostenführerschaft }\end{array}$ & $\begin{array}{l}\text { - Produktentwicklung mit } \\
\text { Kunden } \\
\text { - Internationale Vermark- } \\
\text { tung } \\
\text { - Preis-/Leistungsverhältnis } \\
\text { - Investitionen Marketing- } \\
\text { maßnahmen } \\
\text { - Intensive Kundenbezie- } \\
\text { hungen } \\
\text { - Kostenführerschaft }\end{array}$ \\
\hline Management & $\begin{array}{l}\text { - Professionelles Management durch starke Führungs- } \\
\text { persönlichkeiten; Eigentümerführung } \\
\text { - Leistungsorientierte Unternehmenskultur; Vorgabe } \\
\text { ambitionierter Zielsetzungen } \\
\text { - Konzentration auf die Kernkompetenzen; hoher Eigen- } \\
\text { anteil an der Wertschöpfung bei den Kernkompetenzen } \\
\text { - Hohe Umsetzungskompetenz; schnelle Umsetzung von } \\
\text { Entscheidungen und Projekten } \\
\text { - Internationalisierung der Produktion }\end{array}$ & $\begin{array}{l}\text { - Eigentümerführung } \\
\text { - Leistungsorientierte Un- } \\
\text { ternehmenskultur } \\
\text { - Konzentration auf Kern- } \\
\text { kompetenzen } \\
\text { - Hohe Umsetzungskompe- } \\
\text { tenz } \\
\text { - Internationalisierung der } \\
\text { Produktion }\end{array}$ \\
\hline $\begin{array}{l}\text { Finanzen/ } \\
\text { Betriebswirt- } \\
\quad \text { schaft }\end{array}$ & $\begin{array}{l}\text { - Hohe Finanzkraft/hohe Eigenkapitalquote } \\
\text { - Hohe (Fix-)Kostenflexibilität } \\
\text { - Zentralisierte Organisationsstrukturen; kosteneffizi- } \\
\text { ente, interne Prozesse }\end{array}$ & $\begin{array}{l}\text { - Hohe Finanzkraft } \\
\text { - Kostenflexibilität } \\
\text { - Zentralisierte Organisation }\end{array}$ \\
\hline Mitarbeiter & $\begin{array}{l}\text { - Hohe Identifikation und Motivation der Mitarbeiter mit } \\
\text { den Zielen und Werten des Unternehmens } \\
\text { - Hohe Mitarbeiterqualifikation; hohes Know-how der } \\
\text { Mitarbeiter }\end{array}$ & $\begin{array}{l}\text { - Motivation der Mitarbeiter } \\
\text { - Hohe Mitarbeiterqualifi- } \\
\text { kation }\end{array}$ \\
\hline
\end{tabular}


Zur Messung des Unternehmenserfolgs hat sich das Konzept des Zielansatzes nach Etzioni (1964) etabliert. Dieses nimmt an, dass Unternehmen multidimensionale, individuelle Zielbündel verfolgen und der Erfolg den Zielerreichungsgrad (Effektivität) bzw. die Erreichung eines bestimmten Zielniveaus mit möglichst geringem Input (Effizienz) darstellt (Baumgarth und Evanschitzky 2009, 243).

Als Erfolgsindikatoren für die vorgelegte Studie wurden entsprechend den Studien zum Industrial Organisation-Ansatz die Eigenkapitalrentabilität sowie als zusätzlicher PerformanceIndikator das Umsatzwachstum der Unternehmen sowie der relative Marktanteil als Zielansatz gewählt. Wir schließen uns damit den meisten bestehenden empirischen Studien zur Erfolgswirksamkeit von strategischer Planung an, die ebenfalls finanzielle Indikatoren als abhängige Variable verwendeten (vgl. Hall und Weiss 1967; Pearce et al. 1987; Rue und Ibrahim 1998; Schmalensee 2007). Außerdem wurde die Anzahl der direkten Wettbewerber erhoben.

\section{Stichprobe}

Die Stichprobe der Studie setzte sich zu einem Großteil aus Unternehmen mit bis zu 499 Beschäftigten (74,7 \%) und bis zu 50 Mio. Euro Umsatz (67,8 \%) zusammen. Die genauen Verteilungen lassen sich den Abbildungen 1 und 2 entnehmen.

Die Stichprobe der Studie setzte sich unterschiedlich aus den mittelstandsrelevanten Branchen Handel, Handwerk, Dienstleistung und Industrie zusammen. Erwartungsgemäß waren dabei die Branchen Industrie (43,6 \%) und Dienstleistung (30,9 \%) stärker, der Handel und das Handwerk mit 18,2 \% bzw. 4,5\% bei den befragten mittelständischen Unternehmen schwächer vertreten. Die Analyse der Eigentümerstruktur ergab, dass 59,2 \% der Unternehmen inhabergeführt waren, 14,5\% der Unternehmen waren im Besitz externer Gesellschafter und jeweils 21 \% gehörten einer größeren Unternehmensgruppe oder einem Konzern an. Lediglich 5,3 \% der befragten Unternehmen waren zum Befragungszeitpunkt börsennotiert. Hinsichtlich der Eigentümerstruktur ist anzumerken, dass diese Variable in einer dritten Erhebungswelle nicht erhoben wurde, weshalb der Stichprobenumfang für diese Auswertung lediglich bei $N=80$ lag.

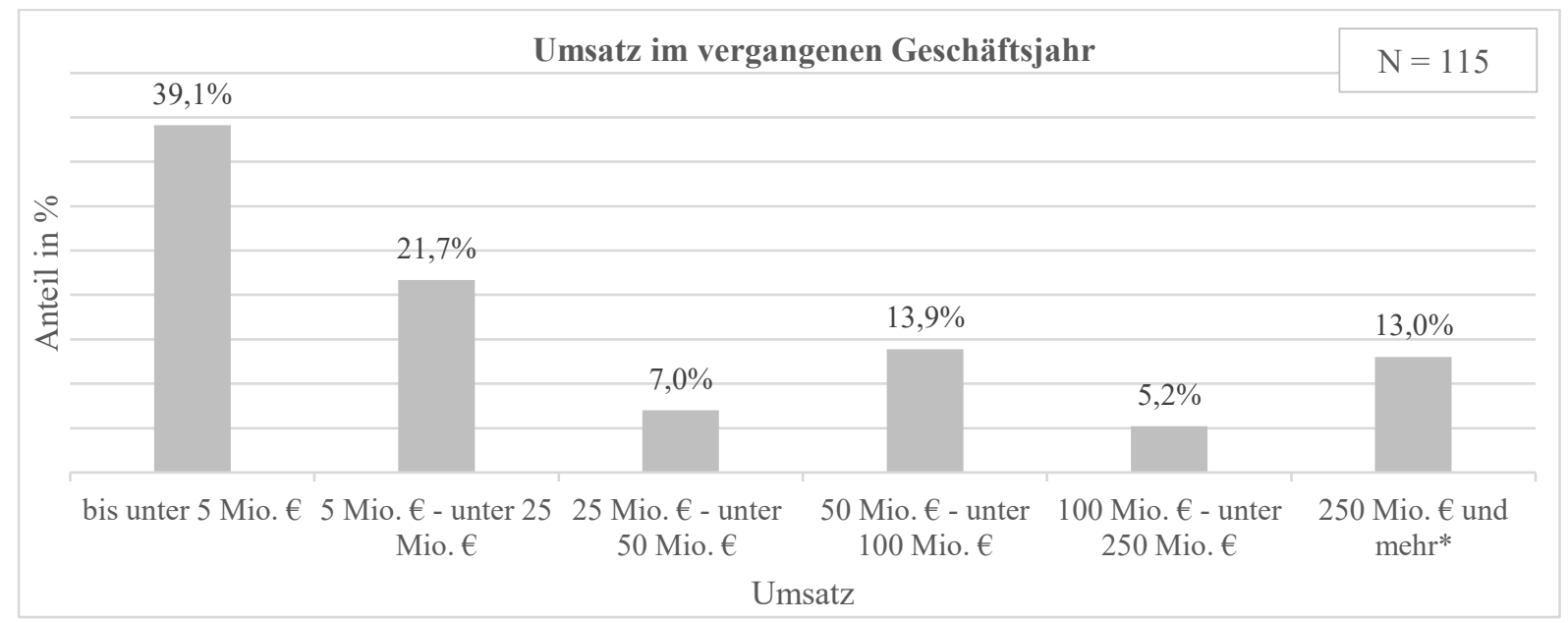

Abbildung 1: Beschreibung der Stichprobe hinsichtlich des Umsatzes.

* maximaler Umsatz bis zu 400 Millionen Euro. 


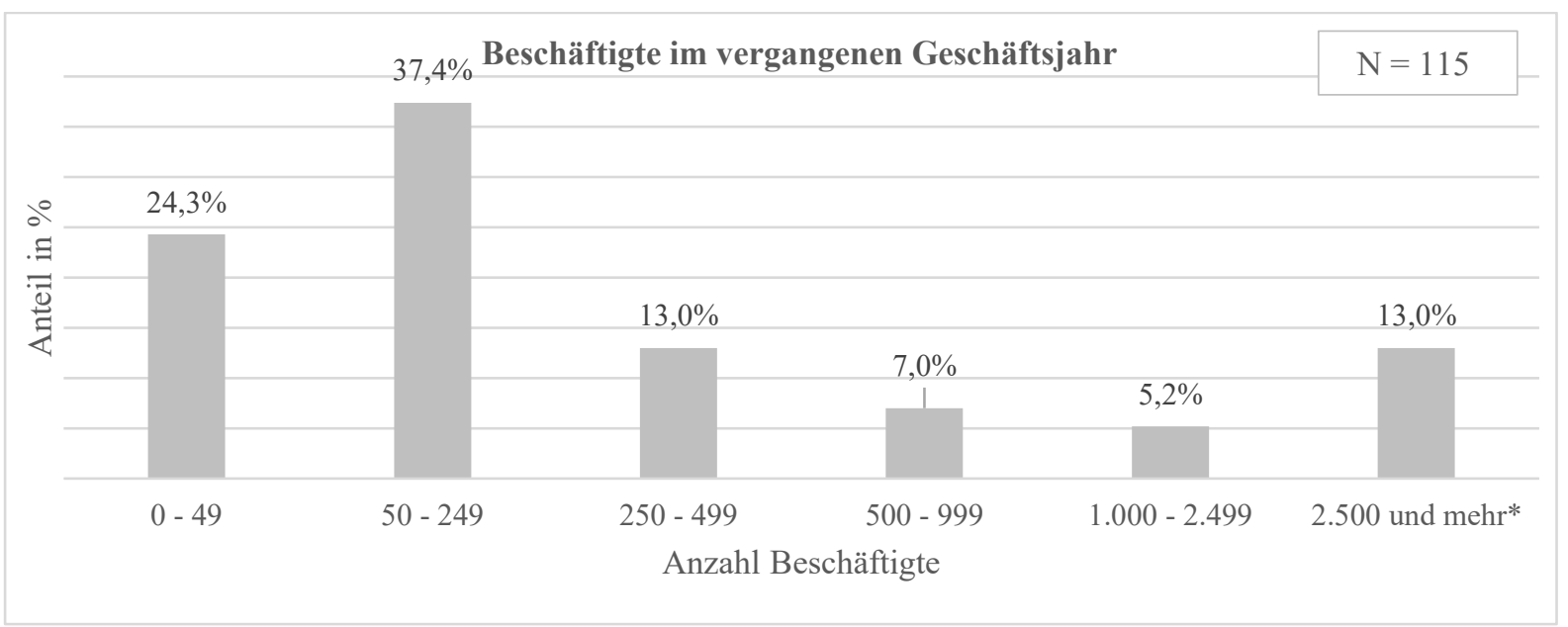

Abbildung 2: Beschreibung der Stichprobe hinsichtlich der Beschäftigtenanzahl * maximal bis zu 5.000 Mitarbeitern.

\section{Ergebnisse}

Zur Analyse der Frage, welche Bedeutung mittelständische Unternehmen, die nicht den HC zugeordnet werden können, den von Simon erhobenen Erfolgsfaktoren in der Praxis zumessen, wird zunächst die Bedeutung der Erfolgsfaktoren für die Unternehmen im Allgemeinen betrachtet, bevor ein Vergleich zwischen erfolgreichen und nicht-erfolgreichen Unternehmen hinsichtlich der Bedeutungseinschätzung der Erfolgsfaktoren gezogen wird. Die Darstellung der Ergebnisse erfolgt dabei zunächst rein deskriptiv. Abschließend werden die Ergebnisse mithilfe einer multivariaten Clusteranalyse weiter analysiert, um tiefergehende Erkenntnisse zu generieren.

\section{Bedeutung der Erfolgsfaktoren im Allgemeinen}

Ein Ziel der vorliegenden Untersuchung war es, herauszufinden, welche Bedeutung die befragten mittelständischen Unternehmen den von Simon und anderen Forschern identifizierten Erfolgsfaktoren zumessen. Zunächst wurde daher in der Auswertung der Befragung die Bedeutung der Erfolgsfaktoren analysiert. Zur besseren Orientierung wurden die erhobenen Erfolgsfaktoren - basierend auf Plausibilitätsüberlegungen - zusätzlich verschiedenen Oberkategorien (Produkt/Services, Marketing/Vertrieb, Management, Betriebswirtschaft/Finanzen, Mitarbeiter) zugeordnet, die im Folgenden aber nicht weiter statistisch untersucht wurden. Ein Überblick über die Bedeutungszumessung findet sich in Abbildung 3.

Bei der Einzelanalyse der Erfolgsfaktoren wird deutlich, dass folgende fünf Erfolgsfaktoren eine dominierende Stellung eingenommen haben:

1. Intensive Kundenbeziehungen $(M=4,61, S D=0,54)$

2. Produkt-/Servicequalität $(M=4,47, S D=0,58)$

3. Motivation der Mitarbeiter $(M=4,45, S D=0,55)$

4. Mitarbeiterqualifikation $(M=4,30, S D=0,59)$

5. Hohe Umsetzungskompetenz $(M=4,80, S D=0,72)$ 


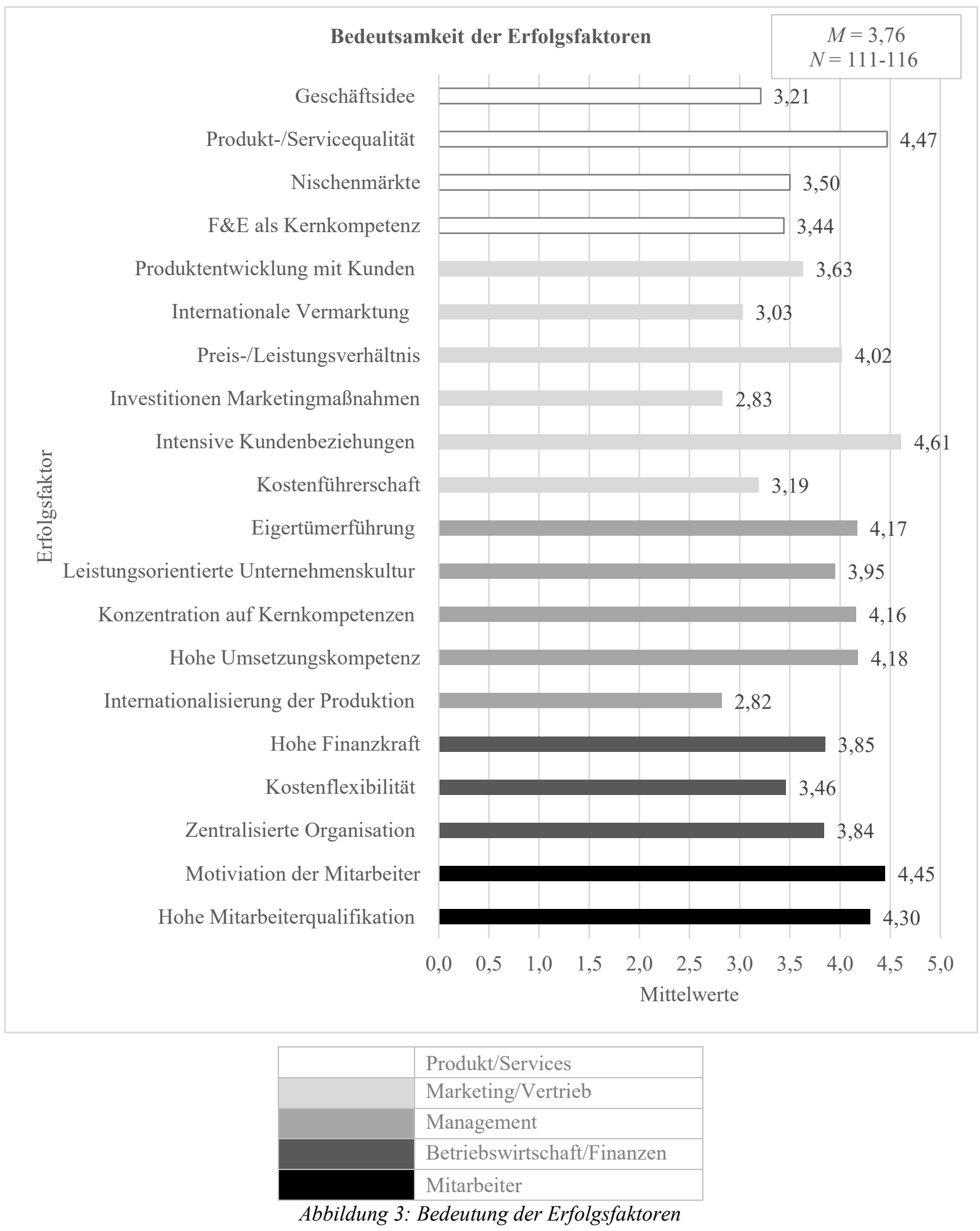

Diesen Ergebnissen zufolge ist jede Oberkategorie - mit Ausnahme der Kategorie der Betriebswirtschaft/Finanzen - mit mindestens einem Erfolgsfaktor unter den Top Fünf vertreten. Dabei setzten die befragten Unternehmen auf dem Markt bevorzugt auf eine intensive Kooperation mit den Kunden, die in einer hohen, kundenorientierten Produkt-/und Servicequalität mündet. 
Die fünf Faktoren mit der geringsten Bedeutung für den Unternehmenserfolg waren:

1. Internationalisierung der Produktion $(M=2,82, S D=1,33)$

2. Hohe Investitionen in Marketingmaßnahmen $(M=2,83, S D=0,89)$

3. Internationale Vermarktung der Unternehmensleistungen $(M=3,03, S D=1,45)$

4. Kostenführerschaft $(M=3,19, S D=0,95)$

5. Einzigartige Geschäftsidee $(M=3,21, S D=1,19)$

Die befragten mittelständischen Unternehmen sahen in der Gesamtbewertung keine wesentlichen Felder zur Erlangung von Wettbewerbsvorteilen bei der Internationalisierung der Produktion, der internationalen Vermarktung sowie den Investitionen in das Marketing. Auch die Einzigartigkeit der Geschäftsidee wurde nicht als besonders bedeutend für den Unternehmenserfolg empfunden.

\section{Bedeutung der Erfolgsfaktoren für erfolgreiche und weniger erfolgreiche}

\section{Unternehmen}

Zur Untersuchung der Grundfrage der Erfolgsfaktorenforschung, ob es Unterschiede in der Bedeutung und Bewertung von Erfolgsfaktoren zwischen erfolgreichen und weniger erfolgreichen Unternehmen gibt, wurde in der Folge eine Analyse der Bedeutungsbeimessung in Abhängigkeit von einem Erfolgskriterium durchgeführt. Zur Messung der individuellen Performance der Unternehmen wurde als abhängige Variable die Eigenkapitalrentabilität sowie als zusätzlicher Performance-Indikator das Umsatzwachstum mittels Selbsteinschätzung erhoben. Die Unternehmen gaben demnach an, ob sie ihre eigene Position im Vergleich zur Branche, als über-, gleich oder unterdurchschnittlich bewerten. Unternehmen, die nach eigener Einschätzung eine überbranchendurchschnittliche Performance zeigten, wurden demnach von denen, die eine branchendurchschnittliche oder unterdurchschnittliche Performance - gemessen an der Eigenkapitalrendite - zeigten, getrennt. Abbildung 4 zeigt die Bewertung der Erfolgsfaktoren durch die Unternehmen nach Unternehmenserfolg.

Zusammengefasst lässt sich aus dieser Analyse erkennen, dass die Einschätzung der relevanten Erfolgsfaktoren zwischen beiden analysierten Gruppen relativ ähnlich war. Allerdings ergaben sich im Detail doch einige interessante Unterschiede, wenn man sich erneut die Erfolgsfaktoren mit der höchsten und niedrigsten Bedeutung im Vergleich anschaut (Tabelle 3 und Tabelle 4).

Betrachtet man zunächst die Rangfolge der wichtigsten Erfolgsfaktoren in beiden Gruppen, so ist zu erkennen, dass beide Gruppen ähnliche Erfolgsfaktoren als besonders bedeutend herausstellen, wenn auch die genaue Rangfolge leicht divergiert. So sehen sowohl erfolgreiche als auch gleich und weniger erfolgreiche Unternehmen in intensiven Kundenbeziehungen, der Motivation der Mitarbeiter und einer hohen Produkt-/Servicequalität wichtige Erfolgsfaktoren. Eine hohe Mitarbeiterqualifikation liegt in beiden Gruppen absolut gesehen auf dem vierten Rang, wobei erfolgreiche Unternehmen diesen Faktor als etwas wichtiger wahrnahmen als die Gruppe der gleich bzw. weniger erfolgreichen Unternehmen. Lediglich beim Faktor des fünften Rangs gab es Unterschiede. So sahen erfolgreiche Unternehmen eine Eigentümerführung als wichtigen Erfolgsfaktor an, wohingegen ein gutes Preis-/Leistungsverhältnis bei den gleich/weniger erfolgreichen Unternehmen diesen Rang einnahm. 
Manuskript - Hidden Champions: Eine empirische Analyse für mittelständische Unternehmen

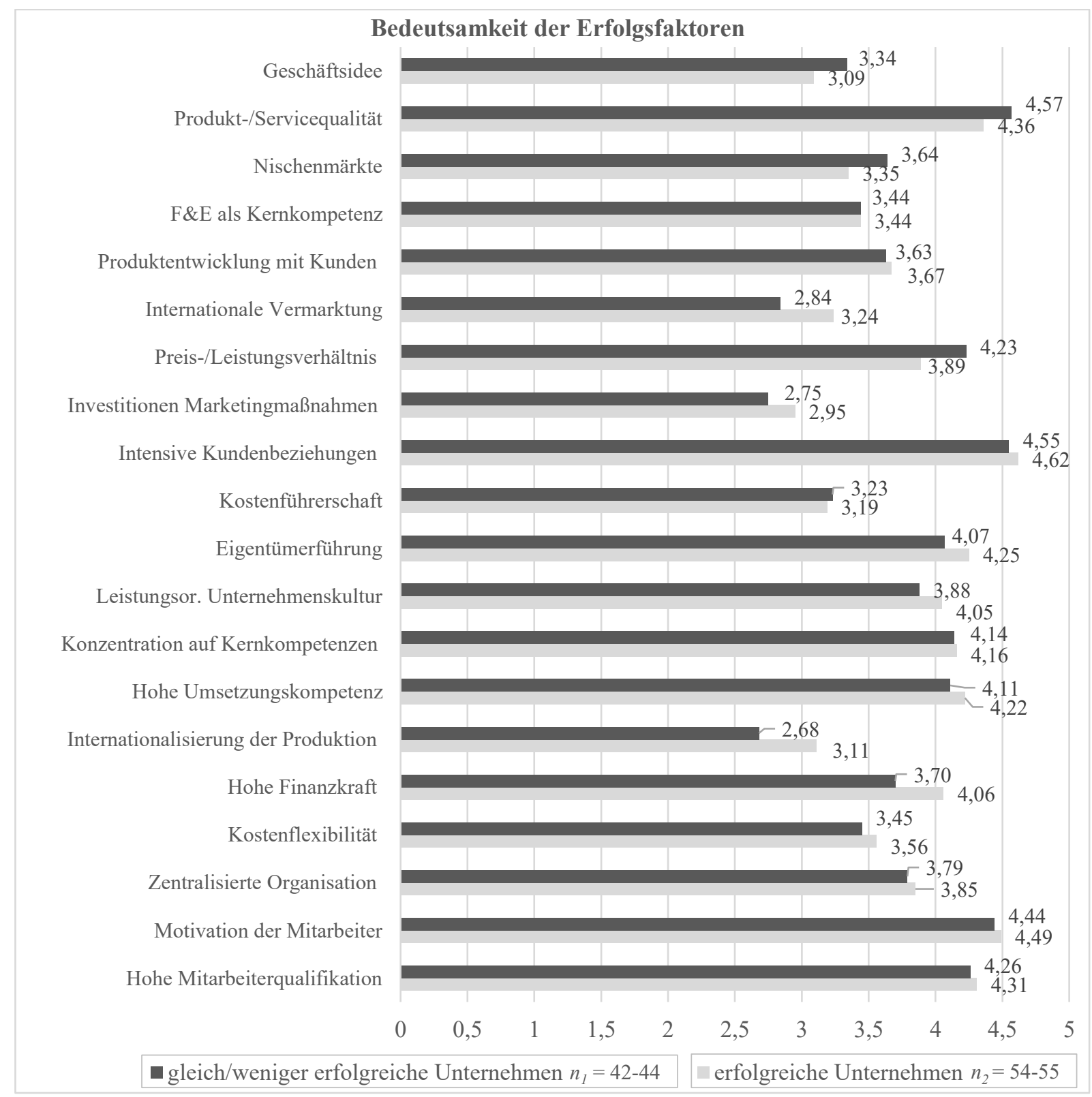

Abbildung 4: Bedeutung der Erfolgsfaktoren in Abhängigkeit vom Unternehmenserfolg

Tabelle 3: Erfolgsfaktoren mit der höchsten Bedeutung

\begin{tabular}{llrrlrr}
\hline Rang & Erfolgreich & $\boldsymbol{M}$ & $\boldsymbol{S D}$ & Gleich/weniger erfolgreich & $\boldsymbol{M}$ & $\boldsymbol{S D}$ \\
\hline 1 & Intensive Kundenbeziehungen & 4,62 & 0,56 & Produkt-/Servicequalität & 4,57 & 0,59 \\
2 & Motivation der Mitarbeiter & 4,49 & 0,57 & Intensive Kundenbeziehungen & 4,55 & 0,55 \\
3 & Produkt-/Servicequalität & 4,36 & 0,52 & Motivation der Mitarbeiter & 4,44 & 0,50 \\
4 & Hohe Mitarbeiterqualifikation & 4,31 & 0,57 & Hohe Mitarbeiterqualifikation & 4,26 & 0,62 \\
5 & Eigentümerführung & 4,25 & 0,65 & Preis-/Leistungsverhältnis & 4,23 & 0,57 \\
\hline
\end{tabular}

Anmerkung: erfolgreiche vs. gleich/weniger erfolgreiche Unternehmen der Stichprobe 
Tabelle 4: Erfolgsfaktoren mit der geringsten Bedeutung

\begin{tabular}{llrrlrr}
\hline Rang & Erfolgreich & $\boldsymbol{M}$ & $\boldsymbol{S D}$ & Gleich/weniger erfolgreich & $\boldsymbol{M}$ & $\boldsymbol{S D}$ \\
\hline 20 & $\begin{array}{l}\text { Investitionen Marketingmaß- } \\
\text { nahmen }\end{array}$ & 2,95 & 0,93 & $\begin{array}{l}\text { Internationalisierung der Pro- } \\
\text { duktion }\end{array}$ & 2,68 & 1,24 \\
19 & Geschäftsidee & 3,09 & 1,22 & $\begin{array}{l}\text { Investitionen Marketingmaßnah- } \\
\text { men }\end{array}$ & 2,75 & 0,84 \\
18 & $\begin{array}{l}\text { Internationalisierung der Pro- } \\
\text { duktion }\end{array}$ & 3,11 & 1,34 & Internationale Vermarktung & 2,84 & 1,46 \\
17 & Kostenführerschaft & 3,19 & 0,99 & Kostenführerschaft & 3,23 & 0,83 \\
16 & Internationale Vermarktung & 3,24 & 1,39 & Geschäftsidee & 3,34 & 1,20 \\
\hline
\end{tabular}

Anmerkung: erfolgreiche vs. gleich/weniger erfolgreiche Unternehmen der Stichprobe

Die Ergebnisse decken sich nahezu vollständig mit den Ergebnissen zur Bedeutung der Erfolgsfaktoren für die Gesamtstichprobe (Abbildung 3). Lediglich die hohe Umsetzungskompetenz findet sich weder bei erfolgreichen noch bei den gleich/weniger erfolgreichen Unternehmen auf den ersten fünf Rängen.

Hinsichtlich der Erfolgsfaktoren von geringerem Gewicht fällt auf, dass sowohl erfolgreiche als auch gleich/weniger erfolgreiche Unternehmen die gleichen Faktoren auf den letzten fünf Rängen sehen, allerdings variiert die Reihenfolge erneut. Während erfolgreiche Unternehmen Investitionen in Marketingmaßnahmen als am wenigsten bedeutend für den Unternehmenserfolg ansahen, war bei den gleich/weniger erfolgreichen Unternehmen der Erfolgsfaktor der Internationalisierung der Produktion auf dem letzten Rang.

Insgesamt können drei der fünf Erfolgsfaktoren der letzten Ränge der Oberkategorie Marketing/Vertrieb zugeordnet werden. Dieses Ergebnis deckt sich mit den Ergebnissen der allgemeinen Betrachtung der Bedeutung der Erfolgsfaktoren (Abbildung 3). Somit gab es keine nennenswerten Unterschiede zwischen erfolgreichen und gleich/weniger erfolgreichen Unternehmen im Vergleich zur Gesamtstichprobe hinsichtlich der Einschätzung der Bedeutung der Erfolgsfaktoren.

Gesamtheitlich betrachtet zeigte sich, dass sich die nach Einschätzung der Unternehmen fünf wichtigsten bzw. unwichtigsten Erfolgsfaktoren in Abhängigkeit des Unternehmenserfolgs kaum divergieren. Auf Basis eines t-Tests, ergab sich dementsprechend, dass sich die beiden Gruppen lediglich hinsichtlich zweier Faktoren statistisch signifikant voneinander unterschieden (gutes Preis-/Leistungsverhältnis der Produkte/Services $\left(M_{1}=3,89, S D_{1}=0,86 ; M_{2}=4,23\right.$, $\left.S D_{2}=0,57 ; t(92)=2,35, p=.022\right)$ sowie hohe Finanzkraft/Eigenkapitalquote $\left(M_{1}=4,06, S D_{1}\right.$ $\left.\left.=0,79 ; M_{2}=3,70, S D_{2}=0,74 ; t(95)=-2,28, p=.025\right)\right)$. Während also weniger/gleich erfolgreiche Unternehmen ein gutes Preis-/Leistungsverhältnis als wichtig erachteten, maßen erfolgreiche Unternehmen einer hohen Finanzkraft eine höhere Bedeutung für den Unternehmenserfolg zu. Auch im Vergleich der beiden Teilstichproben zur Gesamtstichprobe lassen sich nur minimale Unterschiede auf deskriptiver Ebene erkennen. 


\section{Cluster-Analyse}

Zur weiteren Analyse der Erfolgsfaktoren der Hidden Champions für mittelständische Unternehmen wurde die Bedeutung der Erfolgsfaktoren mithilfe des multivariaten, strukturentdeckenden Verfahrens der Clusteranalyse genauer untersucht. Dabei sollte die Frage beantwortet werde, ob in dem vorliegenden Datensatz verschiedene Gruppen identifiziert werden können, die sich hinsichtlich ihrer Bewertung der Erfolgskriterien unterscheiden und so unterschiedliche Geschäftsmodelle salient machen.

Zunächst wurde der Datensatz mithilfe des Single-Linkage-Verfahrens auf Ausreißer untersucht. Da keine auffälligen Werte gefunden worden sind, wurde anschließend das Ward-Verfahren für die Clusterbildung genutzt. Nach Abwägung theoretischer Überlegungen, der Analyse des Dendrogramms und anderer statistischer Kriterien wurde schließlich eine Zwei-Clusterlösung als präferierte Lösung ausgewählt. Die zwei gefundenen Cluster weisen Stichprobengrößen von $n_{1}=87$ und $n_{2}=23$ auf, somit konnten insgesamt 110 der 116 Datensätze für die Cluster-Analyse genutzt werden. Die sechs ausgeschlossenen Datensätze wiesen bei der Bewertung der Erfolgsfaktoren zu viele fehlende Werte auf.

\section{a. Bewertung der Erfolgsfaktoren}

Insgesamt zeigte sich, dass die beiden Cluster die Bedeutung der Erfolgskriterien im Durchschnitt unterschiedlich einschätzten. Abbildung 5 gibt eine Übersicht über die durchschnittliche Bewertung der Bedeutung der Erfolgskriterien durch die zwei Cluster und zeigt, dass sich die zwei Segmente in einigen Aspekten wesentlich unterschieden, während sie bei anderen nahezu identisch waren oder Unterschiede nur marginal ausfielen. In Tabelle 5 sind die Ergebnisse des t-Tests dargestellt, der ergab, dass die Unterschiede zwischen den Clustern in der Bewertung bei zwölf von 20 Erfolgsfaktoren statistisch signifikant waren. Die größten statistisch signifikanten Unterschiede zwischen den Clustern bestanden in der Bewertung der folgenden Erfolgskriterien:

- Starke internationale bzw. globale Vermarktung der Unternehmensleistung $\left(M_{1}=3,55, S D_{1}=1,22 ; M_{2}=1,35, S D_{2}=0,65 ; t(67)=11,74, p=.000\right)$

- $\quad F \& E$ als Kernkompetenz $\left(M_{1}=3,82, S D_{1}=0,96 ; M_{2}=2,09, S D_{2}=1,20 ; t(108)=7,28, p=.000\right)$

- Internationalisierung der Produktion $\left(M_{1}=3,20, S D_{1}=1,23 ; M_{2}=1,52, S D_{2}=0,73 ; t(59)=8,31, p=.000\right)$

- $\quad$ Einzigartige Geschäftsidee $\left(M_{1}=3,54, S D_{1}=1,07 ; M_{2}=1,96, S D_{2}=0,77 ; t(47)=8.06, p=.000\right)$

- Konzentration auf Nischenmärkte $\left(M_{1}=3,64, S D_{l}=1,08 ; M_{2}=2,74, S D_{2}=0,96 ; t(108)=3,65, p=.000\right)$

Auf Basis dieser Unterschiede wird Cluster 1 als „Internationale Innovatoren“ betitelt und Cluster 2 als „Nationale Traditionalisten“. 
Tabelle 5: Ergebnisse des t-Tests zwischen den zwei Clustern „Internationale Innovatoren “ (Cluster 1) und „Nationale Traditionalisten “(Cluster 2)

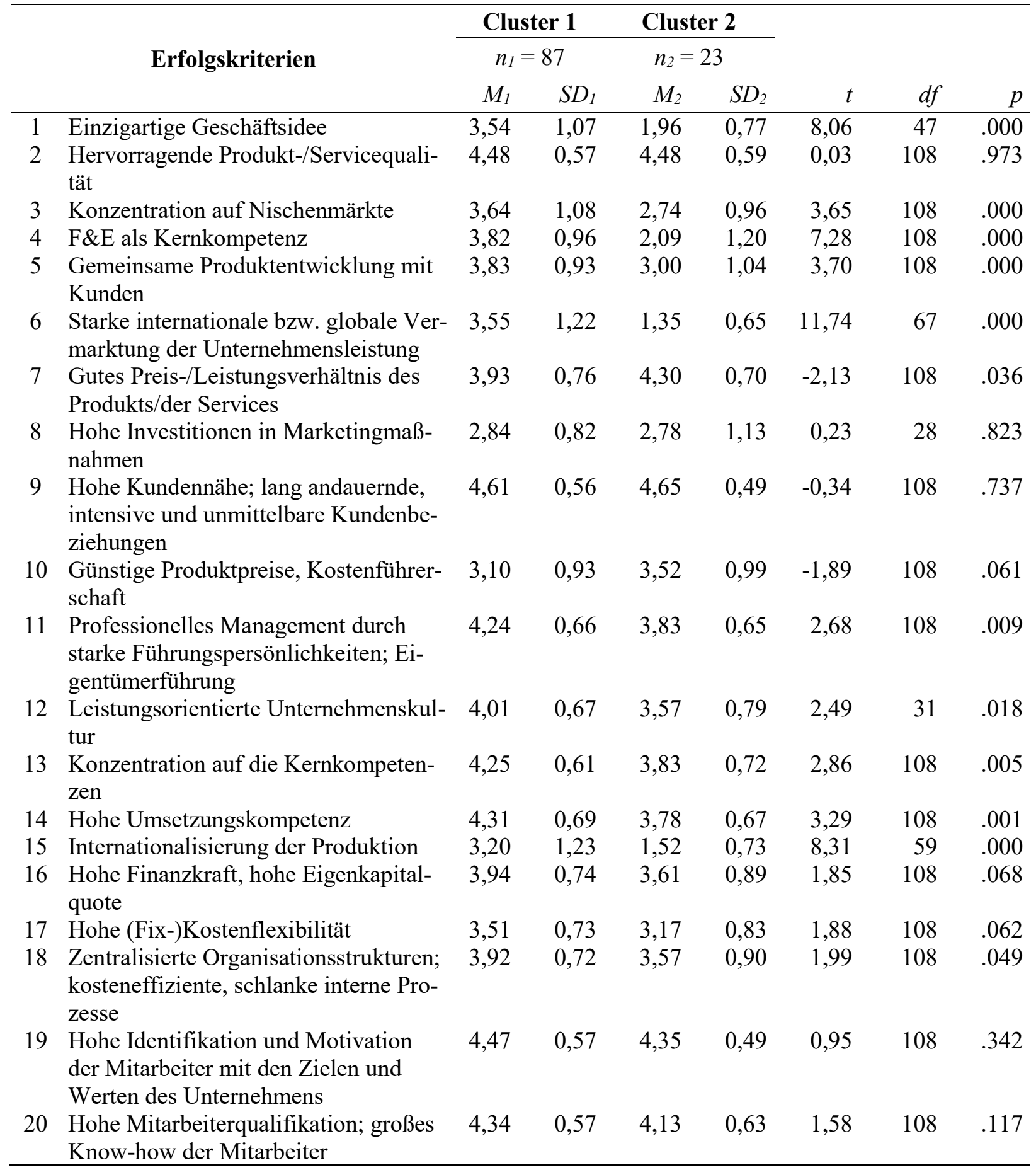


Durchschnittliche Bewertung der Erfolgskriterien nach Cluster

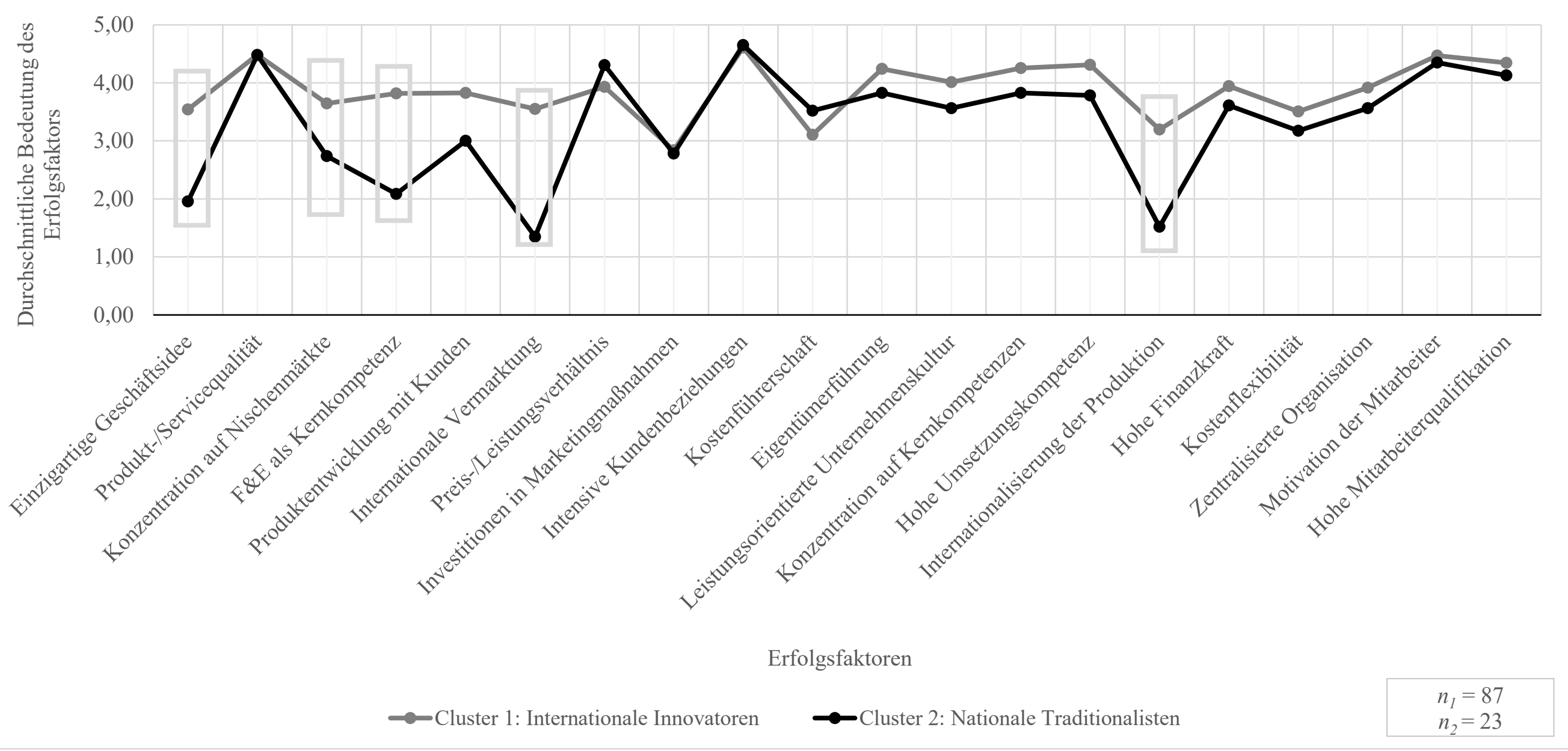

Abbildung 5: Durchschnittliche Bewertung der Erfolgskriterien nach Cluster.

Die Bedeutung nimmt mit aufsteigenden Werten zu. $1=$ sehr gering $/$ keine, $5=$ sehr hoch. 
Cluster 1, die ,internationalen Innovatoren“, wies in allen fünf Erfolgskriterien eine höhere durchschnittliche Bewertung auf, während Cluster 2, die „,nationalen Traditionalisten“ die Erfolgsfaktoren als weniger bedeutsam einschätzte. Die „internationalen Innovatoren“ (1) zeichneten sich damit vor allem durch eine hohe Einschätzung der Wichtigkeit der Internationalisierung, von Innovation bzw. Einzigartigkeit sowie eines eng definierten Kerngeschäfts bzw. eng definierten Markts aus. Somit zeigte sich, dass Unternehmen in Cluster 1 einige wichtige der in der Literatur (Simon 1996; 2007; Blommen und Bothe 2007; Simon 2012; Welter et al. 2014) identifizierten Erfolgsfaktoren von Hidden Champions ebenfalls als sehr bedeutsam für ihren eigenen Erfolg einschätzten. Infolgedessen könnte man die Unternehmen, die den ,internationalen Innovatoren“ (1) angehören, hinsichtlich der Beurteilung der Erfolgsfaktoren tendenziell eher als „Hidden Champions“ identifizieren, als die Unternehmen in Cluster 2, die den „nationalen Traditionalisten“ (2) angehören.

Cluster 2 beurteilte lediglich den Erfolgsfaktor „Gutes Preis-/Leistungsverhältnis des Produkts/der Services“ $\left(M_{1}=3,93, S D_{1}=0,76 ; M_{2}=4,30, S D_{2}=0,70 ; t(108)=-2,13, p=.036\right)$ statistisch signifikant als bedeutsamer als Cluster 1. Dies deckt sich allerdings erneut mit den Befunden der Literatur, da erfolgreiche Hidden Champions eher eine Qualitäts- als eine Preisführerschaftsstrategie verfolgen. Somit liegt es nahe, dass die ,internationalen Innovatoren“ (1) hier einen signifikant geringeren Wert aufweisen. Auch die Bewertung der Bedeutung des Erfolgsfaktors „Günstige Produktpreise, Kostenführerschaft“ fiel für die „,nationalen Traditionalisten" (2) höher aus als für Cluster $1\left(M_{1}=3,93, M_{2}=4,30, p=.036\right)$. Da dieser Unterschied jedoch nicht statistisch signifikant war, stellt die zuvor angestellte These lediglich eine Interpretationsmöglichkeit dar, die nicht sicher bestätigt werden kann.

b. Beschreibung der Cluster anhand demografischer Merkmale

Neben den Unterschieden in der Bewertung der Erfolgskriterien können die Cluster ebenfalls anhand ihrer demografischen Merkmale beschrieben werden, sofern die befragten Unternehmen Angaben hierzu machten.

\section{Branche}

Bei den ,internationalen Innovatoren“ (1) waren knapp über die Hälfte der Unternehmen (51,2 $\%)$ in der Industriebranche tätig, gefolgt von der Dienstleistungsbranche (26,2 \%). Handel (14,3 $\%)$ und Handwerk (6,0 \%) waren in diesem Cluster weniger vertreten. Im zweiten Cluster, den „nationalen Traditionalisten“, arbeiteten mit 40,0\%, die meisten Unternehmen in der Dienstleistungsbranche, gefolgt von der Handelsbranche mit 35,0 \%. Demgegenüber waren nur 20,0 $\%$ der Unternehmen in der Industriebranche tätig, wohingegen keines der Unternehmen im Handwerk tätig war. Zwei Unternehmen in Cluster 1 (2,4 \%) bzw. ein Unternehmen in Cluster $2(5,0 \%)$ ordneten sich einer anderen Branche als der Handels-, Industrie-, Handwerks- oder Dienstleistungsbranche zu. Während also bei den ,internationalen Innovatoren“ die meisten Unternehmen in der Industrie tätig waren, ließ sich das Hauptgeschäft bei der Mehrheit der „nationalen Traditionalisten“ der Dienstleistungs- bzw. Handelsbranche zuordnen.

\section{Mitarbeitende}

Bezüglich der Anzahl der Mitarbeitenden im letzten Geschäftsjahr zeigten sich zwischen den beiden Clustern keine großen Unterschiede. Die meisten befragten Unternehmen beider Cluster 
beschäftigten zwischen 50 und 249 Mitarbeitende (Cluster 1: 34,9\%, Cluster 2: 52,2\%). Auf dem zweiten Platz waren ebenfalls in beiden Clustern Unternehmen mit 0 bis 49 Mitarbeitenden (Cluster 1: 22,1\%, Cluster 2: 21,7 \%). In der Gruppe der ,internationalen Innovatoren“ (1) schlossen sich auf dem dritten Platz Unternehmen mit 250 bis 499 Mitarbeitenden (15,1\%) an. Große Unternehmen mit mehr als 2.500 Mitarbeitenden waren in beiden Clustern prozentual gesehen ähnlich stark vertreten (Cluster 1: 14,0 \%, Cluster 2: 13,0 \%). Die übrigen Größenklassen waren in beiden Clustern zu kleineren Anteilen vertreten. Insgesamt lässt sich festhalten, dass bei den ,internationalen Innovatoren“ (1) die Mehrheit (72,1 \%) der befragten Unternehmen bis zu 499 Mitarbeitende beschäftigte, während der Großteil (73,9 \%) der Unternehmen der ,nationalen Traditionalisten“ (2) in Hinblick auf die Mitarbeiterzahl etwas kleiner war und bis zu 249 Mitarbeitende beschäftigte.

\section{Eigentümerstruktur}

In Bezug auf die Eigentümerstruktur existierten ebenfalls keine entscheidenden Unterschiede zwischen den Clustern. Über die Hälfte der Unternehmen in beiden Clustern waren inhabergeführte Unternehmen oder Familienunternehmen (Cluster 1: 54,5 \%, Cluster 2: 66,7 \%), wobei die inhabergeführten Unternehmen in Cluster 2 stärker vertreten waren. Auch in Hinblick auf andere Eigentumsverhältnisse unterschieden sich die beiden Cluster nicht maßgeblich. So befanden sich in Cluster 1, den ,internationalen Innovatoren“, 20 \% im Besitz unternehmensexterner Einzelgesellschafter, jeweils 9,1 \% waren Konzernunternehmen oder Teil einer Unternehmensgruppe. Der kleinste Anteil der Unternehmen in Cluster 1 war börsennotiert (7,3 \%).

Im zweiten Cluster, den „nationalen Traditionalisten“, waren 20,0 \% Konzernunternehmen und 13,3 \% Teil einer Unternehmensgruppe. Keines der Unternehmen in Cluster 2 war börsennotiert oder im Besitz unternehmensexterner Einzelgesellschafter.

\section{Wettbewerber}

Bei der Wettbewerbsintensität in den gebildeten Clustern lassen sich aufgrund der durchgeführten Analyse interessante, strategiekonforme Unterschiede in Hinblick auf die Wettbewerbsvorteile der Unternehmen in der Hinsicht erkennen, dass Unternehmen im Cluster ,internationale Innovatoren" aufgrund ihrer strategiespezifischen Wettbewerbsvorteile einem wesentlich geringeren Wettbewerbsdruck ausgesetzt sind als die Unternehmen des Clusters „nationale Traditionalisten".

Im Detail gab es bei den ,internationalen Innovatoren“ (1) bei der Anzahl der direkten Wettbewerber im wesentlichen Markt eine Konzentration bei ca. 4 bis 10 Wettbewerbern (45,3\%). Etwas mehr als ein Viertel der Unternehmen (27,9\%) in Cluster 1 sahen sich mit ca. 1 bis 3 Wettbewerbern konfrontiert und circa ein weiteres Viertel (26,7\%) gaben an, mehr als 10 Wettbewerber zu haben. Im Gegensatz dazu, sahen sich bei den „,nationalen Traditionalisten“ (2) die meisten Unternehmen mit mehr als 10 Wettbewerbern (43,5\%) konfrontiert. Knapp dahinter mit 39,1 \% lagen Unternehmen, die ca. 4 bis 10 Wettbewerber sahen. Nur 17,4 \% der Unternehmen in Cluster 2 gaben an, ca. 1 bis 3 Wettbewerber zu haben.

Bei der Anzahl der direkten Wettbewerber im wesentlichen Markt ließ sich somit erkennen, dass die ,internationalen Innovatoren“ (1), die ebenfalls eine einzigartige Geschäftsidee und die Konzentration auf Nischenmärkte als bedeutsame Erfolgskriterien bewerteten, prozentual 
gesehen im Vergleich zu den „,nationalen Traditionalisten“(2) auch weniger Wettbewerber hatten.

Damit lässt sich die Strategie der ,internationalen Innovatoren“ auch passend mit der Wettbewerbsmatrix nach Porter (2014) untermauern bzw. erklären. So erzielten die Unternehmen, die als ,internationale Innovatoren“ identifiziert wurden, einen strategischen Vorteil, indem sie stärker auf die Einzigartigkeit ihrer Produkte und Dienstleitungen fokussierten, denn auf einen Kostenvorsprung. Durch die weitere Konzentration auf Teilmärkte, ließ sich zudem oftmals eine Nischenstrategie erkennen. Je höher die Differenzierung der Produkte bzw. Dienstleistungen ist, desto geringer ist auch die Wettbewerbsintensität, da die angebotenen Waren nur schwer zu substituieren und sehr gut auf die spezifischen Kundenbedürfnisse abgestimmt sind (z. B. Porter 2014; Woeckener 2014). Diese zentrale Erkenntnis lässt sich auch in den erhobenen Daten beobachten.

\section{Umsatz und Umsatzwachstum}

Auch in Bezug auf das Erfolgskriterium „Umsatzwachstum“ zeigen sich Unterschiede zwischen den beiden Clustern. Sofern die befragten Unternehmen eine Angabe zum Umsatz gemacht haben, erwirtschafteten je ca. 50 \% der Unternehmen der ,internationalen Innovatoren“ (1) bis bzw. über 50 Mio. Euro Umsatz (50,9 \% zu 49,1\%). In Cluster 2, den ,nationalen Traditionalisten“, machten 68,8 \% der Unternehmen weniger als 50 Mio. Euro Umsatz und 31,3 $\%$ mehr als 50 Mio. Euro Umsatz im letzten Geschäftsjahr.

In Hinblick auf das durchschnittliche Umsatzwachstum der letzten fünf Jahre im Vergleich zum Branchendurchschnitt schätzten die Hälfte der Unternehmen in Cluster 1 ihr Umsatzwachstum über dem Branchenschnitt (50,0 \%) ein, während nur 26,1 \% der Unternehmen des zweiten Clusters angaben, dass ihr Umsatzwachstum als überdurchschnittlich zu bewerten sei. Die Mehrzahl der Unternehmen in Cluster 2 ordnete ihr Umsatzwachstum lediglich auf Höhe des Branchendurchschnitts (52,2 \%) ein. Bei den ,internationalen Innovatoren“ (1) schätzten 36,0 \% der Unternehmen ihr Umsatzwachstum auf Höhe des Branchenschnitts ein und die wenigsten Unternehmen gaben an, unter dem Branchenschnitt (4,7 \%) zu liegen, wohingegen 13,0 $\%$ des Clusters $2 \mathrm{ihr}$ Umsatzwachstum als unterdurchschnittlich einschätzten.

So zeigte sich insgesamt, dass die „internationalen Innovatoren“ (1) durchschnittlich gesehen nicht nur mehr Umsatz erzielten, sondern auch subjektiv eher das Gefühl hatten, ein Umsatzwachstum über dem Branchendurchschnitt erzielt zu haben. Die meisten Unternehmen des Clusters der „nationalen Traditionalisten“ schätzten ihr Umsatzwachstum eher durchschnittlich ein und erzielten weniger als 50 Mio. Euro Umsatz im Jahr.

\section{Eigenkapitalrentabilität}

Hinsichtlich der durchschnittlichen Eigenkapitalrentabilität der letzten fünf Jahre im Vergleich zum Branchendurchschnitt zeigte sich ein ähnliches Bild wie beim Umsatzwachstum. Die meisten der ,internationalen Innovatoren“ (1) schätzten ihre durchschnittliche Eigenkapitalrentabilität höher ein als die des Branchendurchschnitts (Cluster 1: 51,8 \%), gefolgt von Unternehmen, die sich selbst auf Höhe des Branchendurchschnitts einordneten (Cluster 1: 32,9 \%). Bei den „nationalen Traditionalisten“ (2) schätzten je 39,1 \% der Unternehmen ihre Eigenkapitalrentabilität der letzten fünf Jahre auf Höhe oder über dem Branchendurchschnitt ein. Die wenigsten 
Unternehmen in beiden Clustern bewerteten ihre Eigenkapitalrentabilität unter dem Branchendurchschnitt (Cluster 1: 2,4\%, Cluster 2: 8,7\%). In beiden Clustern gab es aber Befragte, die dazu keine Angabe machten (Cluster 1: 12,9\%, Cluster 2: 13,0 \%).

Damit war der Anteil an Unternehmen, die ihre Eigenkapitalrentabilität als branchenüberdurchschnittlich einschätzen, ähnlich wie beim Umsatzwachstum, bei den ,internationalen Innovatoren“ (1) wesentlich höher als bei den „nationalen Traditionalisten“ (2). Betrachtet man die finanziellen Indikatoren gesamtheitlich, wird deutlich, dass die Unternehmen des ersten Clusters, die als ,,internationale Innovatoren“ identifiziert wurden, über eine höhere Finanzkraft verfügen, als die „nationalen Traditionalisten“. Da die „internationalen Innovatoren“ viele Merkmale der Hidden Champions aufweisen und vermehrt eine Nischenstrategie verfolgen, die in einer geringeren Wettbewerbsintensität resultiert, ist diese Erkenntnis kaum überraschend.

\section{Fazit}

Die Ergebnisse der Untersuchung zeigen, dass die befragten mittelständischen Unternehmen die Bedeutung der von Simon und anderen Autoren postulierten Erfolgsfaktoren von Hidden Champions anerkennen und als grundsätzlich wichtig für ihren eigenen Erfolg einschätzen. Am wichtigsten erachteten die Befragten den Aufbau intensiver Kundenbeziehungen, eine gute Produkt-/Servicequalität, eine hohe Motivation und Qualifikation der Mitarbeiter und eine hohe Umsetzungskompetenz. Bei der Analyse der Bedeutung der Erfolgsfaktoren in Abhängigkeit des Unternehmenserfolgs konnten lediglich marginale Unterschiede zwischen erfolgreichen und gleich/weniger erfolgreichen Unternehmen, gemessen am Branchendurchschnitt, auf deskriptiver Ebene festgestellt werden. Allerdings konnte dieses Bild durch einen t-Test bestätigt werden. Die Unterschiede zwischen erfolgreichen und weniger erfolgreichen Unternehmen waren bis auf zwei Ausnahmefälle statistisch nicht signifikant. Statistische Signifikanz konnte einzig bei Bedeutungsunterschieden in der Bewertung der Faktoren Preis-/Leistungsverhältnis und hohe Finanzkraft/Eigenkapitalquote beobachtet werden. So setzten erfolgreiche Unternehmen weniger auf Preiswettbewerb und maßen gleichzeitig einer hohen Finanzkraft eine höhere Bedeutung zu als gleich/weniger erfolgreiche Unternehmen. Insgesamt konnten aber keine oder nur minimale Unterschiede in der Bedeutungseinschätzung der Erfolgsfaktoren in Abhängigkeit des Unternehmenserfolgs beobachtet werden.

Anders war dies in der Clusteranalyse. Ziel war es, Gruppen im Datensatz zu identifizieren, die die Erfolgsfaktoren in sich möglichst homogen und im Vergleich zu anderen Gruppen möglichst heterogen beantwortet haben, um so herauszufinden, ob es Geschäftsmodelle in mittelständischen Unternehmen gibt, die sich die Strategien der Hidden Champions zunutze machen. Mithilfe dieses Verfahrens konnten zwei Cluster definiert werden. Diese Cluster unterschieden sich statistisch signifikant in der Bewertung von zwölf der insgesamt 20 Erfolgsfaktoren. Unternehmen des ersten Clusters, die als ,internationale Innovatoren“ identifiziert wurden, maßen insbesondere den Faktoren einer starken internationalen bzw. globalen Vermarktung der Unternehmensleistung, F\&E als Kernkompetenz, der Internationalisierung der Produktion, einer einzigartigen Geschäftsidee und der Konzentration auf Nischenmärkte eine höhere Bedeutung für ihren Unternehmenserfolg zu als die Unternehmen in Cluster 2, den ,nationalen Traditionalisten“. Zudem zeichneten sich die Unternehmen der ,,internationalen Innovatoren“ des ersten 
Clusters durch höhere Einschätzungen hinsichtlich der Erfolgsmaßstäbe Umsatz, Umsatzwachstum sowie Eigenkapitalrentabilität im Vergleich zu den „nationalen Traditionalisten“ aus.

Gleichzeitig gab die Mehrheit der Unternehmen der ,internationalen Innovatoren“ an, über vier bis zehn Wettbewerber zu verfügen, wohingegen die Mehrheit der Unternehmen der ,nationalen Traditionalisten“ über zehn Wettbewerber hatte. Durch die höhere Differenzierung der „internationalen Innovatoren“ konnte auch in Einklang mit der Literatur (z. B. Porter 2014) eine geringere Wettbewerbsintensität im Vergleich mit den „nationalen Traditionalisten“ beobachtet werden. Während außerdem über die Hälfte der Unternehmen der ,internationalen Innovatoren“" in der Industriebranche tätig waren, ordneten sich die meisten Unternehmen der „,nationalen Traditionalisten“ der Dienstleistungsbranche zu.

All diese Faktoren zusammennehmend, konnten die Unternehmen des Clusters der „internationalen Innovatoren" als Unternehmen identifiziert werden, die die klassischen Strategien der Hidden Champions einsetzen und die vor allem in der innovationsaffinen Industriebranche und häufig auf Nischenmärkten tätig sind. Diese Ergebnisse gehen damit mit den Resultaten der Analysen von Holz et al. (2020) einher, die ebenfalls Hidden Champions empirisch untersuchten. Sie stellten fest, dass sich der Unternehmenstyp der Hidden Champions vor allem durch Internationalisierungs- und F\&E-Aktivitäten auszeichnet. Weiterhin ist er geprägt vom Willen, den eigenen Marktanteil auf Grundlage der Kernkompetenzen zu maximieren, sowie bestrebt, technologische Prozesse zu verbessern. So werden Marktnischen bewusst besetzt und global ausgebaut. Auch wiesen die Ergebnisse von Holz et al. (2020) darauf hin, dass ,,produzierende Unternehmen größere Chancen habe, zur Gruppe der Hidden Champions zu gehören als Unternehmen aus dem Dienstleistungs- und Distributionssektor“ (S.1). Im Vergleich mit der faktisch orientierten Identifikation von Erfolgsfaktoren bei Hidden Champions nach Simon, zeigt die vorliegende Studie, wie Mittelständler die Bedeutung der Erfolgsfaktoren für ihren eigenen Unternehmenserfolg subjektiv perzipieren. Im Ergebnis konnte zudem insgesamt festgestellt werden, dass die Erfolgsfaktoren in ihrer Wichtigkeit unterschiedlich gewichtet werden können.

Zwar geben die Analysen der vorliegenden Studie nun Aufschluss darüber, welche erfolgskritischen Faktoren der Hidden Champions Mittelständler im Allgemeinen als wichtiger erachten, dennoch darf nicht außer Acht gelassen werden, dass sich daraus keine kausalen Schlüsse ziehen lassen. Ob also eine Konzentration auf die von den ,internationalen Innovatoren“ bevorzugten Erfolgsfaktoren zwangsläufig zu höherer Rendite oder Umsatzwachstum führt, muss in weiteren Studien untersucht werden.

Weiterhin muss in zukünftigen Studien geprüft werden, was die unterschiedliche Gewichtung in der Bedeutungszumessung für das tatsächliche Handeln der Unternehmen und das Erreichen der Erfolgsfaktoren bedeutet. Aus Plausibilitätsüberlegungen heraus, liegt es nahe anzunehmen, dass Unternehmen, die bestimmten Erfolgsfaktoren eine höhere Bedeutung für ihren eigenen Unternehmenserfolg beimessen auch ihr strategisches Handeln auf diese Faktoren ausrichten. $\mathrm{Ob}$ und inwiefern die Bedeutungszumessung das tatsächliche Handeln beeinflusst, muss in zukünftigen Studien gezielt näher beleuchtet werden.

Darüber hinaus muss beachtet werden, dass insbesondere die ökonomischen Maße lediglich anhand von Selbsteinschätzungen auf relativer und nicht auf absoluter Ebene erhoben wurden. Diese Vorgehensweise des Key Informant Approach impliziert ggf. einen Key Informant Bias, der die Validität der Einschätzungen sowohl der unabhängigen als auch abhängigen Variablen 
(Erfolgsgrößen) beeinflussen kann (Klarmann 2008, 124 ff.). Jedoch zeigen methodische Korrelationsstudien (z. B. Dess und Robinson 1984; Pearce et al. 1987; Dawes 1999; Wall et al. 2004; alle zitiert nach Baumgarth und Evanschitzky 2009) eine mittlere bis hohe und überwiegend signifikante Korrelation zwischen subjektiv erhobenen Urteilen und objektiven Kennzahlen, sodass die Selbsteinschätzung im Rahmen des Key Informant Approach durchaus zu validen Aussagen führen kann. Für zukünftige Studien sollten dennoch unterschiedliche Methoden - vor allem auch objektive Daten - zur Datenerhebung herangezogen werden (Klarmann 2008, 230 ff.; Baumgarth und Evanschitzky 2009, 242 f.). Nicht zuletzt hat natürlich - wie oben beschrieben - die Auswahl und Definition des Zielansatzes in der Erfolgsmessung einen Einfluss auf die Bewertung des Unternehmenserfolgs und kann damit auch die Ergebnisse der Erfolgsfaktorenanalyse beeinflussen.

Kritisch muss außerdem angemerkt werden, dass der relativ kleine Stichprobenumfang die generelle Aussagekraft der Untersuchung einschränkt. Hier sollten Studien mit einer größeren Anzahl von Teilnehmern weitere Aufschlüsse bringen. Neben dem kleinen Stichprobenumfang, der keine Generalisierung der Ergebnisse erlaubt, ist als weitere Einschränkung der geografische Fokus der Stichprobe zu nennen. Zur Untersuchung der Bedeutung der Erfolgsfaktoren für mittelständische Unternehmen in Deutschland im Allgemeinen wurden exemplarisch mittelständische Unternehmen mit Sitz in Nordrhein-Westfalen befragt. Dies geschah unter der Annahme, dass sich mittelständische Unternehmen in NRW nicht von Unternehmen, die ihren Sitz anderswo in Deutschland haben, unterscheiden. Da diese Annahme allerdings nicht geprüft ist, ist von einer eingeschränkten Repräsentativität der Stichprobe auszugehen. Zukünftige Untersuchungen sollten daher deutschlandweit mittelständische Unternehmen befragen oder prüfen, ob es messbare, regionale Spezifika gibt.

\section{Literaturverzeichnis}

Baumgarth, C. und Evanschitzky, H. (2009): Erfolgsfaktorenforschung, in: Baumgarth, C., Eisend, M. und Evanschitzky, H. (Hrsg.): Empirische Mastertechniken. Eine anwendungsorientierte Einführung für die Marketing- und Managementforschung, Wiesbaden: Gabler Verlag, $235-261$.

Becker, W., Fischer, S., Staffel, M. und Ulrich, P. (2008): Implementierungsstand von Unternehmensführung und Controlling in mittelständischen Unternehmen. Ergebnisbericht einer empirischen Untersuchung, Bamberg: Deloitte.Mittelstandsinstitut an der Universität Bamberg.

Blommen, D. und Bothe, B. (Hrsg.) (2007) (2007): Die Stars im Mittelstand. Führung und Umsetzung entscheiden, Frankfurt am Main: Frankfurter Allgemeine Buch.

Deimel, K., Ellenberger, M. und Molitor, M. (2017): Strategische Planung in kleinen und mittleren Unternehmen in Deutschland, in: Gadatsch, A., Krupp, A. D. und Wiesehahn, A. (Hrsg.): Controlling und Leadership. Konzepte - Erfahrungen - Entwicklungen, Wiesbaden: Gabler, 91 - 108.

Etzioni, A. (1964): Modern organizations, Englewood Cliffs, N.J.: Prentice-Hall.

Evanschitzky, H. (2003): Konzeptionelle Grundlagen zur Messung des Erfolgs in Dienstleistungsnetzwerken, in: Evanschitzky, H. (Hrsg.): Erfolg von Dienstleistungsnetzwerken, Wiesbaden: Deutscher Universitätsverlag, 53 - 175.

Hall, M. und Weiss, L. (1967): Firm Size and Profitability, in: The Review of Economics and Statistics, 49(3), 319-331. 
Hamel, G. und Prahalad, C. K. (1994): Competing for the future, Boston: Harvard Business School Press.

Holz, M., Schlepphorst, S. und Schlömer-Laufen, N. (2020): Worauf der Erfolg der Hidden Champions beruht, in: Unternehmertum im Fokus(1).

iwd (2019): Hidden Champions. Die Starken aus der zweiten Reihe, [https://www.iwd.de/artikel/hidden-champions-die-starken-aus-der-zweiten-reihe-424550/], abgerufen am 07.12.2020.

Klarmann, M. (2008): Methodische Problemfelder der Erfolgsfaktorenforschung. Bestandsaufnahme und empirische Analysen, 1. Aufl., Wiesbaden: Gabler.

Knyphausen-Aufseß, D. zu (1994): Theorie der strategischen Unternehmensführung. State of the Art und neue Perspektiven, Wiesbaden: Gabler.

Marshall, M. (1996): The key informant technique, in: Family PRactice, 13, 92 - 97.

Möller, K. (2006): Unternehmensnetzwerke und Erfolg: Eine empirische Analyse von Einfluss- und Gestaltungsfaktoren, in: Schmalenbachs Zeitschrift für betriebswirtschaftliche Forschung, 58, $1051-1076$.

Myers, S. (1984): The Capital Structure Puzzle, in: The Journal of Finance, 39(3), 574 - 592.

Nicolai, A. und Kieser, A. (2002): Trotz eklatanter Erfolglosigkeit. Die Erfolgsfaktorenforschung weiter auf Erfolgskurs, in: DBW, 62(6), 579 - 596.

Pearce, J. A., Freeman, E. B. und Robinson, R. B. (1987): The Tenuous Link Between Formal Strategic Planning and Financial Performance, in: Academy of Management Review, 12(4), $658-675$.

Porter, M. E. (2014): Wettbewerbsvorteile. Spitzenleistungen erreichen und behaupten, 12. Aufl., Frankfurt am Main: Campus.

Rockart, J. (1980): Topmanager sollten ihren Datenbedarf selbst definieren, in: Harvard Manager, 2, $45-58$.

von Rosenstiel, L. (2009): Erfolgsfaktor Unternehmenskultur - Beteiligung erfolgreich umsetzen, in: Nerdinger, F. W. und Wilke, P. (Hrsg.): Beteiligungsorientierte Unternehmenskultur. Erfolgsfaktoren, Praxisbeispiele und Handlungskonzepte, Wiesbaden: Gabler, $27-42$.

Rue, L. und Ibrahim, N. A. (1998): The Relationship between Planning Sophistication and Performance in Small Businesses, in: Journal of Small Business Management, 36(4), $45-58$.

Schmalensee, R. (2007): Inter-industry studies of structure and performance, in: Schmalensee, R. und Willig, R. D. (Hrsg.): Handbook of industrial organization, Amsterdam: Elsevier, $951-1009$.

Simon, H. (1996): Die heimlichen Gewinner. Die Erfolgsstrategien unbekannter Weltmarktführer (Hidden champions), Frankfurt am Main: Campus.

Simon, H. (2007): Hidden Champions des 21. Jahrhunderts. Die Erfolgsstrategien unbekannter Weltmarktführer, 1. Aufl., Frankfurt am Main: Campus.

Simon, H. (2012): Hidden Champions - Aufbruch nach Globalia. Die Erfolgsstrategien unbekannter Weltmarktführer, 1. Aufl., Frankfurt am Main: Campus.

Weirauch, O. (2007): Kosten flexibilisieren: Stabile Stückkosten bei jeder Wetterlage, in: Blommen, D. und Bothe, B. (Hrsg.): Die Stars im Mittelstand. Führung und Umsetzung entscheiden, Frankfurt am Main: Frankfurter Allgemeine Buch, 97 - 106.

Welge, M. K., Al-Laham, A. und Eulerich, M. (2017): Strategisches Management. Grundlagen - Prozess - Implementierung, 7. Aufl., Wiesbaden: Springer Gabler. 
Welter, F., Bijedic, T., Brink, S. und Kriwolutzky, S. (2014): Triebwerk des Erfolgs - der deutsche Mittelstand im Fokus, Bonn.

Welter, F., May-Strobl, E., Holz, M., Pahnke, A., Schlepphorst, S., Wolter, H.-J. und Kranzusch, P. (2015): Mittelstand zwischen Fakten und Gefühl, Bonn.

Woeckener, B. (2014): Marktökonomische Grundlagen, in: Woeckener, B. (Hrsg.): Strategischer Wettbewerb. Eine Einführung in die Industrieökonomik, Berlin: Springer Gabler, $1-49$. 